\title{
Effect of Oil-droplet Size on the Oxidation of Microencapsulated Methyl Linoleate
}

\author{
Risa Nakazawa, Motohiro Shima and Shuji Adachi* \\ Division of Food Science and Biotechnology, Graduate School of Agriculture, Kyoto University (Sakyo-ku, Kyoto 606-8502, JAPAN)
}

\begin{abstract}
Effects of oil-droplet size, the weight ratio of oil to wall material and the storage temperature on the oxidation of methyl linoleate microencapsulated with maltodextrin by spray-drying were examined. The oxidation of methyl linoleate was more retarded for the microcapsules prepared from the emulsion having smaller oil droplets. The oxidation was more suppressed for the microcapsules having a lower weight ratio of oil to wall material. The fraction of unoxidized methyl linoleate leveled off after 10- to 15days storage. The level, $Y_{\infty}$, depended on the weight ratio. The dependence of $Y_{\infty}$ on the weight ratio was analyzed based on the percolation theory, and the three-dimensional model of the theory was suitable to express the dependence. The effect of the storage temperature on the oxidation of microencapsulated methyl linoleate was also examined, and the activation energy was evaluated. The value of the energy suggested that the oxidation itself was a rate-limiting step for the oxidation of methyl linoleate encapsulated with maltodextrin.
\end{abstract}

Key words: oxidation; methyl linoleate; nanoemulsion; microencapsulation; spray-drying

\section{INTRODUCTION}

Polyunsaturated fatty acids have physiological functions ${ }^{1,2)}$ but are prone to oxidation even under ambient conditions. Improvement in their oxidative stability is desired for their utilization in the food and pharmaceutical industries. Their microencapsulation with an adequate wall material retards the oxidation. Microencapsulation of a liquid lipid such as polyunsaturated fatty acids and their acylglycerols consists of two steps ${ }^{3}$; one is emulsification of the lipid to produce an $\mathrm{O} / \mathrm{W}$ emulsion, which is a dense solution of a wall material, and another is rapid dehydration of the emulsion to produce microcapsules. Spray-drying is the most popular technique for preparing microcapsules ${ }^{4}$. Factors affecting the oxidative stability of the encapsulated lipid, such as the type of polyunsaturated fatty acid, kind of wall material, and weight ratio of the lipid to wall material, have been examined ${ }^{5-10)}$. We reported that the oxidation proceeded more slowly for the linoleic acid encapsulated with maltodextrin in smaller oildroplets $^{7,8}$. In these reports, the oil-droplet sizes in emulsions were on the orders of submicrometers or micrometers. However, the effect of the oil-droplet size in the emulsion on the oxidative process of a microencapsulated polyunsaturated fatty acid has not been examined for oil droplets of several tens to hundreds of nanometers

In this context, we measured the oxidation processes of methyl linoleate which was encapsulated with maltodextrin from $\mathrm{O} / \mathrm{W}$ emulsions having different oil-droplet sizes and weight ratios of the ester to the wall materials at $50^{\circ} \mathrm{C}$. The processes were kinetically analyzed based on the modified Weibull equation. The feature of the oxidation of methyl linoleate having different weight ratios was analyzed by applying the percolation theor ${ }^{11)}$ to the processes. The effect of the storage temperature on the oxidative stability of the encapsulated methyl linoleate was also examined.

\section{EXPERIMENTAL}

\subsection{Materials}

Methyl linoleate (purity > 95\%) and maltodextrin with a dextrose equivalent of 2 to 5 were purchased from Tokyo Kasei Kogyo (Tokyo, Japan) and Matsutani Chemical Industries (Osaka, Japan), respectively. Butylhydroxytoluene (BHT) and methyl palmitate were purchased from Wako

*Correspondence to: Shuji Adachi, Division of Food Science and Biotechnology, Graduate School of Agriculture, Kyoto University, Sakyoku, Kyoto 606-8502, JAPAN

E-mail: adachi@kais.kyoto-u.ac.jp

Accepted January 29, 2008 (received for review January 21, 2008)

Journal of Oleo Science ISSN 1345-8957 print / ISSN 1347-3352 online

http://www.jstage.jst.go.jp/browse/jos/ 


\section{R. Nakazawa, M. Shima and S. Adachi}

Pure Chemical Industries (Osaka, Japan). Decaglycerol monolaurate was supplied by Sakamoto Chemical Industries (Osaka, Japan).

\subsection{Microencapsulation of methyl linoleate}

Decaglycerol monolaurate, which was a surfactant, was dissolved in distilled water at a concentration of $30 \%(\mathrm{w} / \mathrm{w})$ at $80^{\circ} \mathrm{C}$, and methyl linoleate was then added to the surfactant solution to produce a concentration of $30 \%(\mathrm{w} / \mathrm{w})$. The mixture was emulsified with a rotor/stator homogenizer (Polytron PT20SK, Kinematica, Lucern, Switzerland) for 3 to $5 \mathrm{~min}$ at $7 \times 10^{4}$ to $1 \times 10^{5} \mathrm{rpm}$ to prepare an $\mathrm{O} / \mathrm{W}$ emulsion. The conditions of homogenization depended on the oil-droplet size in the emulsion. This emulsion was further homogenized by a high-pressure homogenizer (PEL20, Nanomizer, Tokyo, Japan) at 1,000 $\mathrm{kg}$-force $/ \mathrm{cm}^{2}$ three times to prepare an emulsion with smaller oil droplets.

Maltodextrin was dissolved in distilled water under magnetic stirring at $20 \%(\mathrm{w} / \mathrm{w})$. The solution was mixed well with the $\mathrm{O} / \mathrm{W}$ emulsion at the ratios in $\mathrm{g} / \mathrm{g}$ of $600 / 80$, $428.6 / 100$ and $360 / 120$ to yield the weight ratios of oil to wall material of $0.2,0.35$ and 0.5 , respectively, in the microcapsules. The mixture was fed into an LB-8 spray-dryer (Okawara, Tokyo, Japan) at a flow rate of $3.0 \mathrm{~kg} / \mathrm{h}$ through a centrifugal atomizer operated at $3 \times 10^{4} \mathrm{rpm}$. The emulsion in the feed reservoir was magnetically stirred to prevent creaming of the oil droplets. The temperatures of air at the inlet and the outlet were 180 and $90^{\circ} \mathrm{C}$, respectively. The flow rate of the air was $c a .7 .5 \mathrm{~m}^{3} / \mathrm{min}$.

\subsection{Size distributions of microcapsules and oil droplets}

The particle size of the spray-dried microcapsules was measured with a laser diffraction particle size analyzer using methanol as a dispersing solvent. The photos of the microcapsules dispersed in methanol were also taken by a visible light microscope (TMS-F12, Nikon, Tokyo, Japan), and the diameters of $c a .500$ particles were manually measured.

Oil-droplet size distribution in an $\mathrm{O} / \mathrm{W}$ emulsion was measured using a laser diffraction particle size analyzer (SALD-2100, Shimadzu, Kyoto, Japan) and a dynamic light scattering spectrophotometer (DLS-7000, Otsuka Electronics, Osaka, Japan) for the emulsions having the median diameters larger and smaller than $0.1 \mu \mathrm{m}$, respectively, before and after mixing with the maltodextrin solution. The spray-dried microcapsules were dissolved with distilled water to produce an $\mathrm{O} / \mathrm{W}$ emulsion. The oil-droplet size distribution was also measured to examine the change in the oil-droplet size before and after microencapsulation by spray-drying.

\subsection{Oxidation of microencapsulated methyl linoleate}

The spray-dried microcapsules, which contained $c a .5 .3$ $\mathrm{mg}$ of methyl linoleate, were weighed into a $10 \mathrm{~mL}$ vial.
The vial was placed in a $50 \mathrm{~mL}$ vial, in which a saturated lithium chloride solution was included to regulate the relative humidity at $12 \%$. The $50 \mathrm{~mL}$ vial was tightly sealed and stored in a container in the dark at 37,50 or $60^{\circ} \mathrm{C}$. About sixty $50-\mathrm{mL}$ vials were prepared for the measurement of the oxidation process under a specific condition.

At appropriate intervals, three vials were removed from the container to measure the fraction of unoxidized methyl linoleate. A $500 \mu \mathrm{L}$ portion of water, the temperature of which had been regulated to be the same as the storage one, was poured into the $10 \mathrm{~mL}$ vial and vortex-mixed for 3 min in a vortex mixer (E-36, Taitec, Saitama, Japan) to dissolve the microcapsules. The solution $(400 \mu \mathrm{L})$ was added to a mixture of chloroform and methanol (2:1 by vol., 1000 $\mu \mathrm{L}$ ) containing BHT at $5.0 \times 10^{-4} \mathrm{~mol} / \mathrm{L}$ in a microtube, and the mixture was then vortex-mixed again. After centrifugation at $1.5 \times 10^{4} \mathrm{rpm}$ for $5 \mathrm{~min}(\mathrm{MC}-150$, Tomy Seiko, Tokyo, Japan) for separation to the organic and aqueous phases, the lower organic phase $(600 \mu \mathrm{L})$ was pipetted into another microtube. After evaporation of the solvent under reduced pressure, the remainder was dissolved in $300 \mu \mathrm{L}$ of hexane, in which methyl palmitate had been dissolved at a concentration of $3.0 \mathrm{~g} / \mathrm{L}$ as an internal standard in gas chromatographic determination, and agitated. The mixture was centrifuged at $1.5 \times 10^{4} \mathrm{rpm}$ for $1 \mathrm{~min}$ to precipitate the undissolved substrates. The supernatant $(1 \mu \mathrm{L})$ was applied to a GC-2014 gas chromatograph (Shimadzu, Kyoto, Japan) equipped with a hydrogen frame ionization detector. The temperatures of column, injector and detector were 220,250 and $270^{\circ} \mathrm{C}$, respectively. The fraction of unoxidized methyl linoleate was calculated from the ratio in the peak area of unoxidized methyl linoleate to methyl palmitate. Three samples were analyzed for a specific storage time, and the values were averaged.

The pressure in the vial was also measured using a pressure transmitter (VHR3-200A, Valcom, Osaka, Japan) connected to an analog-digital converter (ZAK-01, As One, Osaka, Japan) during the oxidation of encapsulated methyl linoleate and was recorded on a personal computer. The amount of oxygen consumed during the oxidation was estimated assuming that the decrease in the pressure was ascribed to the consumption.

\section{RESULTS AND DISCUSSION}

\subsection{Size distributions of oil droplets and microcapsules}

Figures 1(a) and (b) show the oil-droplet size distribution in the emulsions before and after mixing with the maltodextrin solution to prepare the microcapsules having the weight ratio of methyl linoleate to maltodextrin of 0.2. The oil droplet was smaller for (a) than for (b). The figures also show the oil-droplet distributions for the emulsions which were obtained by dissolving the spray-dried microcapsules 

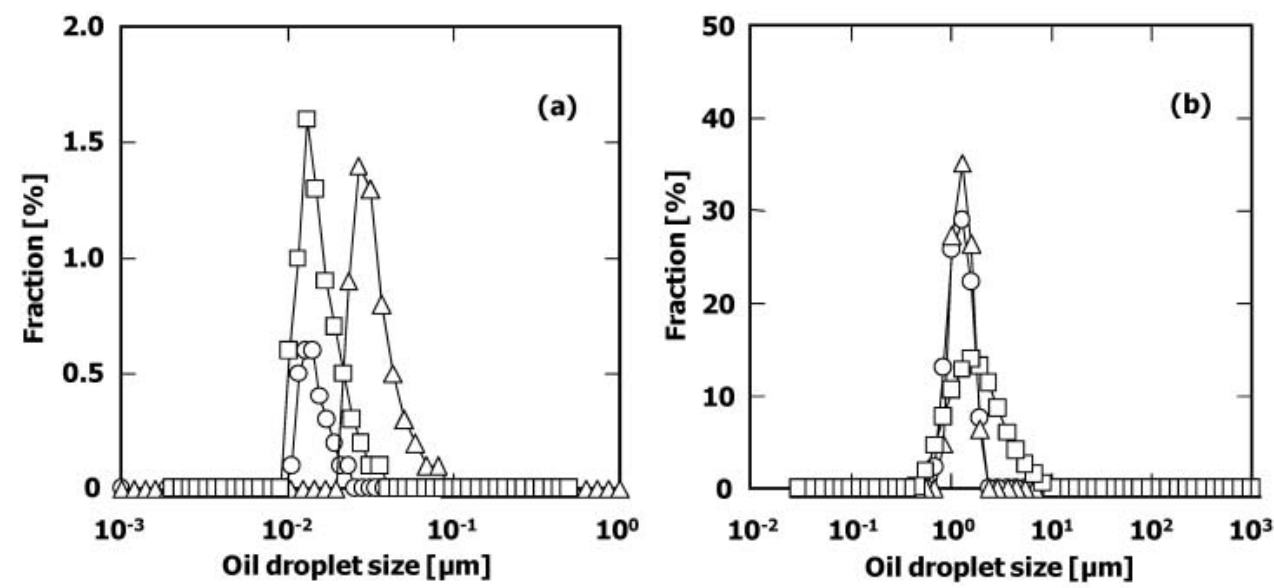

Fig. 1 Distributions of Oil-droplet Size in the Emulsions Having (a) Small and (b) Large Oil Droplets.

Symbols, $\bigcirc, \triangle$ and $\square$, represent the emulsions before and after mixing with maltodextrin solution and the emulsion obtained by dissolving microcapsules. In (a), the fraction ascribed to the emulsifier micelles, which occupied a large fraction, is omitted in the distribution.

with distilled water. As shown in Fig. 1(a), the median diameter of oil droplets before mixing was $0.018 \mu \mathrm{m}$, and it was shifted to $0.031 \mu \mathrm{m}$. This fact indicated that aggregation or coalescence occurred with the addition of maltodextrin. The reason for the aggregation or coalescence remains unclear. A possible explanation is a depression effect by maltodextrin. Because the median diameter of oil droplets in the emulsion obtained by dissolving the microcapsules was $0.026 \mu \mathrm{m}$ and was almost the same as that of the emulsion before mixing with the maltodextrin solution, the aggregated or coalesced oil droplets seemed to be split into small droplets again during atomization in the spraydryer.

For the emulsion having large oil droplets (Fig. 1(b)), the median diameters of the oil droplets in the emulsions before and after mixing with maltodextrin solution were 1.1 and $1.2 \mu \mathrm{m}$, respectively. The median diameter of oil droplets in the emulsion obtained by dissolving the microcapsules was $1.5 \mu \mathrm{m}$. The diameter was slightly enlarged and the distribution was broadened after encapsulation.

Figure 2 shows the particle-size distributions of microcapsules which were prepared from the $\mathrm{O} / \mathrm{W}$ emulsions having a median diameter of oil droplets of $c a .0 .02$ and 1.0 $\mu \mathrm{m}$. The median diameters of the microcapsule prepared from the emulsions having small and large oil droplets were estimated to be 30 and $27 \mu \mathrm{m}$, respectively, by the laser diffraction particle size analyzer and 25 and $24 \mu \mathrm{m}$, respectively, by the optical microscopy. These results indicated that the size of the oil droplets in the emulsion did not affect the size of the microcapsules.

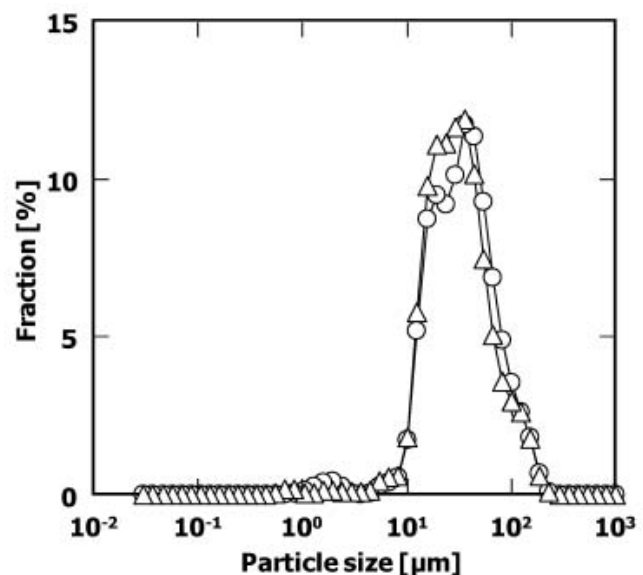

Fig. 2 Size Distributions of Spray-dried Microcapsules. Symbols, $\bigcirc$ and $\triangle$, represent the microcapsules prepared from the emulsions in which the median diameters of oil droplets were $0.02 \mu \mathrm{m}$ and $1 \mu \mathrm{m}$, respectively.

3.2 Oxidation of methyl linoleate in microcapsules having different oil-droplet size and weight ratios

Figures $3(\mathrm{a})$, (b) and (c) show the oxidation processes at $50^{\circ} \mathrm{C}$ of methyl linoleate encapsulated with maltodextrin at weight ratios of the lipid to the wall material of $0.2,0.35$ and 0.5 , respectively. The median diameters of oil droplets in the emulsions before mixing with maltodextrin solution were $0.02,0.06$ and $1.0 \mu \mathrm{m}$. The methyl linoleate was oxidized faster at the higher weight ratio. There seemed to be 


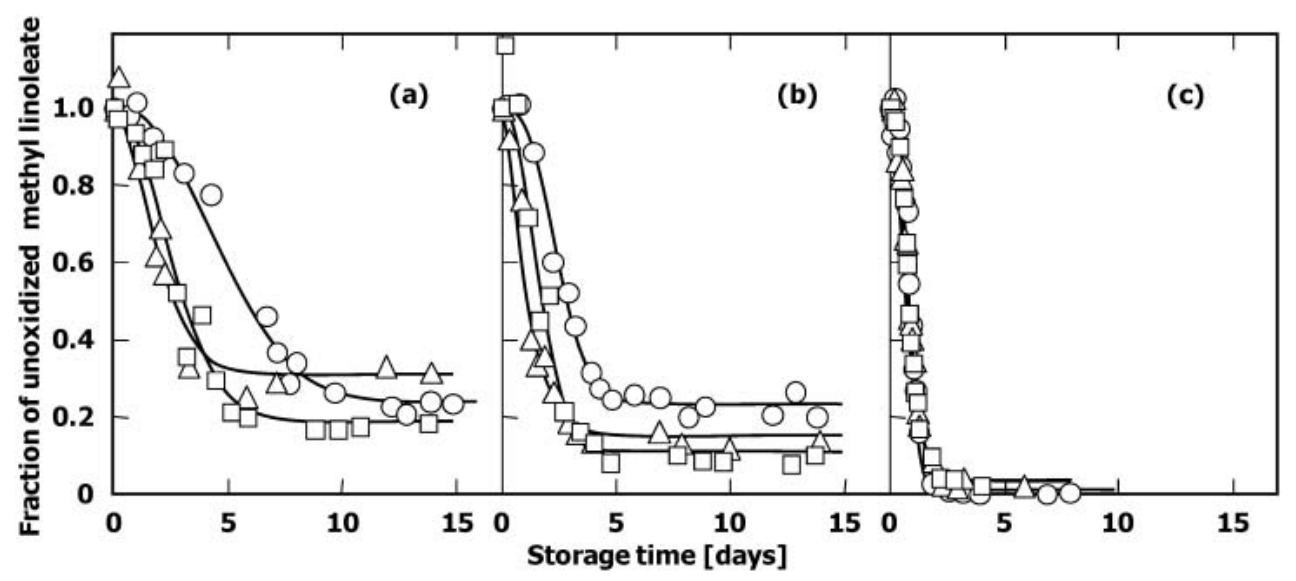

Fig. 3 Oxidation Processes at $50^{\circ} \mathrm{C}$ of Methyl Linoleate Encapsulated with Maltodextrin at the Weight Ratios of the Lipid to the Wall Material of (a) 0.2 , (b) 0.35 and (c) 0.5 . Symbols, $\bigcirc, \triangle$ and $\square$, represent that the median diameters of oil droplets in the emulsions were $0.02,0.6$ and $1 \mu \mathrm{m}$, respectively. The solid curves are drawn based on the modified Weibull equation.

a tendency for methyl linoleate to be oxidized more slowly when the oil-droplet size in the emulsion before mixing the maltodextrin solution was smaller. Except for the microcapsules having the weight fraction of the lipid to wall material of 0.5 , the oxidation of methyl linoleate proceeded extremely slowly or practically leveled off during prolonged storage. This is a feature of the oxidation of encapsulated unsaturated fatty acid. ${ }^{7,8)}$

The change with time of the fraction of unoxidized methyl linoleate in the microcapsule, $Y$, was expressed by the following modified Weibull equation:

$$
\frac{Y-Y_{\infty}}{1-Y_{\infty}}=\exp \left[-(k t)^{n}\right]
$$

where $Y_{\infty}$ is the unoxidized oil fraction at which the oxidation leveled off or practically ceased, $t$ is the storage time, $k$ is a rate constant and $n$ is a shape constant. The $Y_{\infty}$ value was evaluated by averaging the $Y$ values for long storage periods, and the parameters, $k$ and $n$, were then determined to best-fit the experimental results to Eq. (1) using the Solver of Microsoft Excel for Windows. The $Y_{\infty}$ was larger for the microcapsules having the smaller oil droplets. The weight ratio of the lipid to the wall material also affected the $Y_{\infty}$, and $Y_{\infty}$ was higher for the smaller weight ratio.

\subsection{Application of the percolation theory to the oxidation process}

We previously discussed the relationship between the $Y_{\infty}$ and the weight ratio of lipid to wall material based on the percolation theory ${ }^{11)}$ for the oxidation of linoleic acid encapsulated with maltodextrin, pullulan or gum arabic ${ }^{12)}$. The theory also applied to the results obtained in this study.

At first, the relationship was analyzed based on two- and three-dimensional models, in which a microcapsule was assumed to be a square and a cube, respectively, for mathematical ease although the microcapsule is almost spherical. The square and cube were divided into small ones as shown in Fig. 4, and the small squares or cubes occupied by methyl linoleate were randomly allocated to correspond to the volumetric fraction of the lipid within the microcapsule by generating a random number. The lipid located on the periphery of the square and cube began to oxidize, and the oxidation was assumed to propagate to the squares and cubes which faced the oxidized square and cube on the side and in plane, respectively. The isolated squares and cubes were assumed not to be oxidized. The isolated squares or cubes were counted, and the ratio of the number of isolated squares or cubes to that of the whole squares or cubes was considered to respond to the $Y_{\infty}$.

Figure 5 shows the calculated relationships between the $Y_{\infty}$ and the volumetric fraction of methyl linoleate in the microcapsules for the two- and three-dimensional models. The relationships were calculated at various division numbers $N$ on the side and in plane for the two- and threedimensional models, respectively. The larger $N$ value responded to the smaller oil droplets in the microcapsule. For example, an $N$ value of 300 indicates that the oildroplet size is $100 \mathrm{~nm}$ for a microcapsule having a diameter of $30 \mu \mathrm{m}$.

The experimentally obtained $Y_{\infty}$ values are also plotted 
(a)

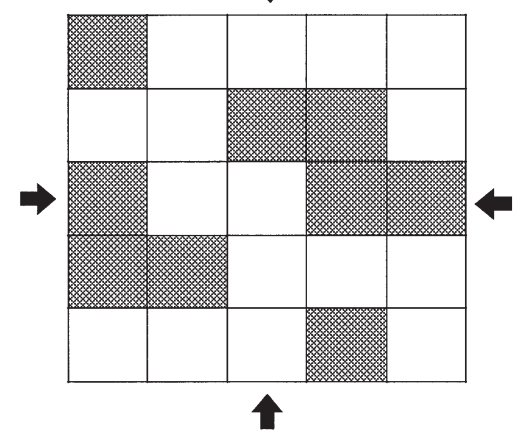

(b)

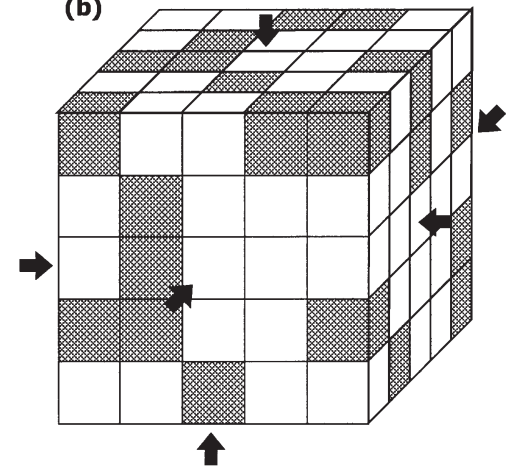

Fig. 4 (a) Two- and (b) Three-dimensional Models for Calculating the $Y_{\infty}$ Value for Various Volumetric Fractions of Methyl Linoleate in a Microcapsule Based on the Percolation Theory. The shaded lattices in (a) and cubes in (b) represent those occupied by methyl linoleate. The lattices or cubes facing the surrounding air are initially oxidized, and those directly contacting the oxidized ones on a side and in plane are oxidized in turn.

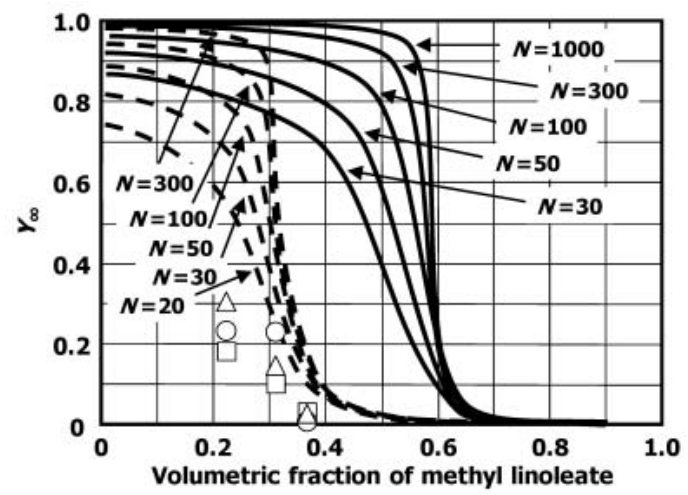

Fig. 5 Relationships between the $Y_{\infty}$ Value and the Volumetric Fraction of Methyl Linoleate Based on the (solid curves) Two- and (broken curves) Three-dimensional Models of the Percolation Theory, and Their Comparison to the Experimental Ones.

Symbols, $\bigcirc, \triangle$ and $\square$, represent the experimental $Y_{\infty}$ values for the microcapsules prepared with the emulsions containing the oil droplets with median diameters of $0.02,0.6$ and $1 \mu \mathrm{m}$, respectively. $N$ in the figure indicates the division number of a side of square or cube. The calculation of $Y_{\infty}$ was repeated 50 to 100 times, depending on $N$, at a volumetric fraction, and the values were averaged. in the figure. The weight ratio of methyl linoleate in a microcapsule was converted to the volumetric fraction assuming that the densities of methyl linoleate and maltodextrin were $0.89 \mathrm{~g} / \mathrm{cm}^{3}$ and $1.5 \mathrm{~g} / \mathrm{cm}^{3}$, respectively. The density of methyl linoleate was quoted from the manufacturer's catalogue and that of maltodextrin was an approximation for saccharides ${ }^{13)}$. Although the $Y_{\infty}$ values are located under the calculated curves, the tendency for the $Y_{\infty}$ value to be steeply decreased near the volumetric fraction of methyl linoleate of 0.3 could be expressed by the threedimensional model. We reported previously that the twodimensional model expressed the characteristics of the oxidation of encapsulated linoleic acid ${ }^{12}$. However, the three-dimensional model was adequate to describe the characteristics of the oxidation of methyl linoleate in the microcapsules in this study. A possible reason for this difference is the difference in the size of the microcapsules between the two studies. In the previous study, microcapsules were prepared by a single-droplet drying method and their diameter was 2 to $3 \mathrm{~mm}$, which was much larger (about 100-fold) than the size of the microcapsules prepared by spray-drying.

The simulation of the relationship reveals that the $Y_{\infty}$ value is larger for a large $N$ value at a specific volumetric fraction of methyl linoleate. As mentioned above, the larger $N$ value indicates the smaller oil droplets in the microcapsules having a specific diameter. Therefore, the size of the oil droplets to be encapsulated is an important factor in the suppression of lipid oxidation, and the oxidation of a lipid would level off or practically cease at a higher level for the microcapsules having smaller oil droplets.

Soottitantawat et al..$^{14,15)}$ reported that some microcapsules have a cavity in their center and that the oil droplets 
were scattered in the shell layer of the dehydrated wall material. We modified the three-dimensional model of the percolation theory so as to simulate the relationship between the $Y_{\infty}$ and the volumetric fraction of methyl linoleate for a spherical microcapsule having a cavity. The cavity would be generated during the drying process due to the evaporation of water and would be filled with air after drying or at least after a long storage in air. Therefore, the methyl linoleate exposed on both the outer and inner surfaces was assumed to be oxidized, and the oxidation was considered to propagate into the layer. Figure 6 shows the relationships between the $Y_{\infty}$ to the volumetric fraction of methyl linoleate for microcapsules having different ratios in the diameter of the inner cavity to the sphere. In this model, the $N$ should be an odd number because of a mathematical reason. The relationships for the full cubic model are also shown in Fig. 6(e). The relationships for the cube were almost the same as those for the full cube because the surface area of a sphere is ca. $80 \%$ of that of a cube when they have the same volume. The tendency for the $Y_{\infty}$ value to decline near the volumetric fraction of 0.3 was common for all the microcapsules having cavities of different sizes and having no cavity, although the volumetric fraction at which the $Y_{\infty}$ value declines shifted to smaller values for smaller $N$ values. The $Y_{\infty}$ value was smaller for the microcapsule having the larger cavity at a specific volumetric fraction of methyl linoleate. This tendency was more significant for a smaller $N$ value.

These calculations suggested that the microencapsulation of emulsion having smaller oil droplets at lower oil content under conditions where no cavity was generated was more favorable for more significantly suppressing the lipid oxidation.

\subsection{Effect of the temperature on the oxidation}

Figure 7 shows the oxidation processes of encapsulated methyl linoleate at 37,50 and $60^{\circ} \mathrm{C}$. The methyl linoleate was encapsulated with maltodextrin at the weight ratio of the lipid to maltodextrin of 0.2 , and the oil-droplet sizes were 0.02 and $1.0 \mu \mathrm{m}$. Oxidation of methyl linoleate in the microcapsules stored at higher temperature was faster, and methyl linoleate in the microcapsules prepared from the emulsion having small oil droplets was more slowly oxidized than that in the microcapsules prepared from the emulsion having large oil droplets irrespective of the storage temperature. Based on Eq. (1), the oxidation processes were analyzed to evaluate the rate and shape constants, $k$ and $n$.

As shown in Fig. 8(a), the temperature dependence of the $k$ value was expressed by the following Arrhenius equation (2):

$$
k=k_{0} \exp (-E / R T)
$$

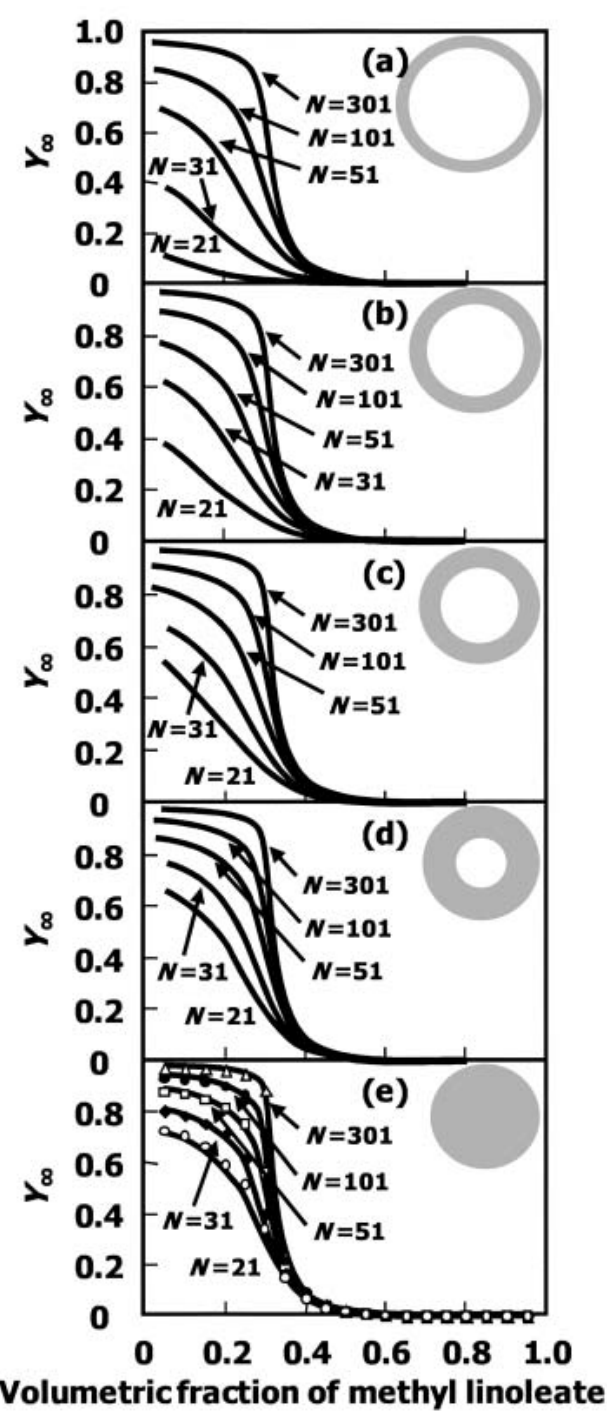

Fig. 6 Relationships between the $Y_{\infty}$ Value and the Volumetric Fraction of Methyl Linoleate in the Microcapsule Having a Cavity.

The ratios in diameter of the cavity to the microcapsule (sphere) are (a) 0.8, (b) 0.7, (c) 0.6 , and (d) 0.4. The curves in (e) are for the full microcapsule, which has no cavity. The volumes of methyl linoleate and maltodextrin were the same in every case. The calculation of $Y_{\infty}$ was repeated 5 to 10 times, depending on $N$, at a volumetric fraction, and the values were averaged. Symbols, $\bigcirc, \diamond, \square, \boldsymbol{O}$ and $\boldsymbol{\Delta}$, represent the $Y_{\infty}$ values for the cubes having the same volumes as the spheres with no cavity. 


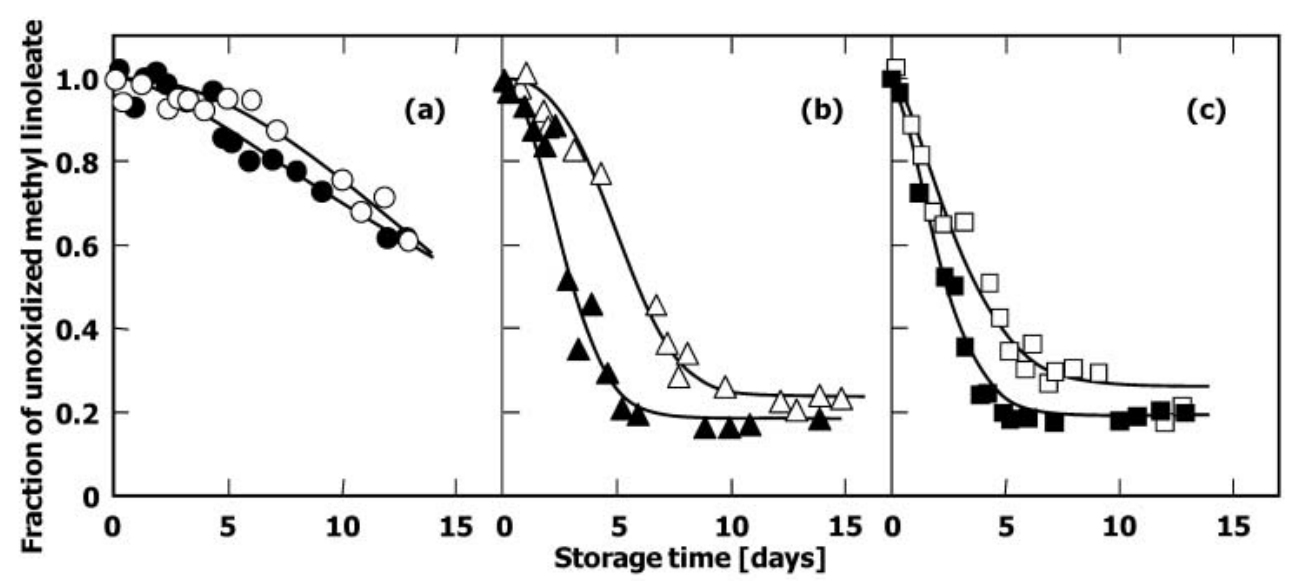

Fig. 7 Oxidation Processes of Methyl Linoleate in the Microcapsules at (a) $37^{\circ} \mathrm{C}$, (b) $50^{\circ} \mathrm{C}$ and (c) $60^{\circ} \mathrm{C}$ and at $12 \%$ Relative Humidity.

Open and closed symbols indicate that the oil-droplet sizes before mixing with maltodextrin were $0.02 \mu \mathrm{m}$ and $1.0 \mu \mathrm{m}$, respectively.

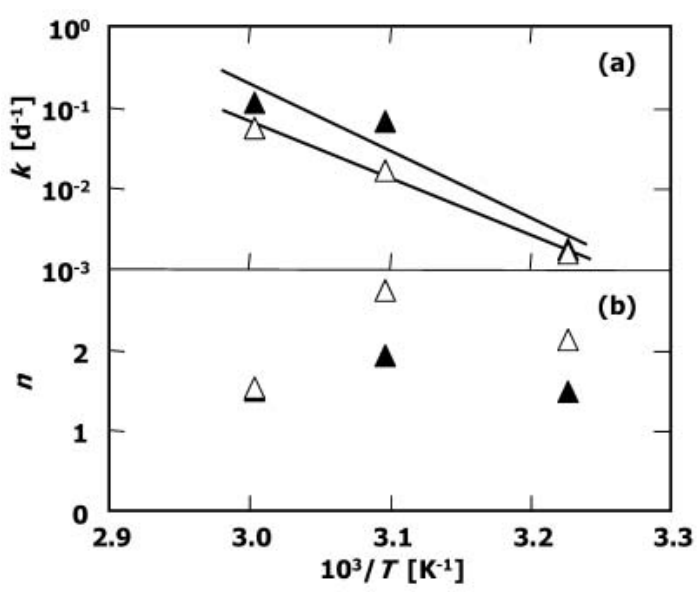

Fig. 8 Temperature Dependences of (a) the Rate Constant $k$ and (b) the Shape Constant $n$ for the Oxidation of Methyl Linoleate Encapsulated with Maltodextrin at the Weight Ratio of the Lipid to the Wall Material of 0.2 .

Open and closed symbols indicate that the oildroplet sizes before mixing with maltodextrin were $0.02 \mu \mathrm{m}$ and $1.0 \mu \mathrm{m}$, respectively.

where $E$ is the activation energy, $k_{0}$ is a frequency factor, $R$ is the gas constant, and $T$ is the absolute temperature. The $E$ values for the microcapsules having the oil droplets of 0.02 and $1.0 \mu \mathrm{m}$ were estimated to be 58 and $70 \mathrm{~kJ} / \mathrm{mol}$, respectively. The values were almost the same as that for the oxidation of methyl linoleate in a bulk system, although the kinetic equation for describing the oxidation was differ- ent from that in this study ${ }^{16}$. This fact suggests that the oxidation itself is a rate-limiting step for the oxidation of the encapsulated lipid and that the diffusion through the dehydrated wall material layer does not significantly affect the oxidation rate.

The $n$ values were larger than unity, indicating the slow oxidation of methyl linoleate during the early stage of storage. Especially, the $n$ values at 50 and $60^{\circ} \mathrm{C}$ were large irrespective to the droplet size of methyl linoleate due to a distinct lag in the oxidation.

\subsection{Stoichiometric relationship between methyl linoleate and oxygen during the oxidation}

Figure 9 shows the stoichiometric relationship between the oxidized methyl linoleate and the oxygen consumed during the oxidation of encapsulated methyl linoleate. The stoichiometric coefficient could be approximated to be unity in the first half of the oxidation, but it remarkably increased in the latter half of the oxidation as the oxidation proceeded. The relationship for methyl linoleate in a bulk system at $40^{\circ} \mathrm{C}^{17}$ is also shown in the figure and was the same as those for the encapsulated methyl linoleate. This fact indicated that encapsulation of methyl linoleate with maltodextrin did not affect its oxidation mechanism.

\section{ACKNOWLEDGMENTS}

This study was financially supported as a Food Nanotechnology Project by the Ministry of Agriculture, Forestry, and Fisheries, Japan. The surfactant, SYGlyster ${ }^{\circledR}$ ML-750, was supplied by Sakamoto Yakuhin Kogyo (Osaka, Japan). 


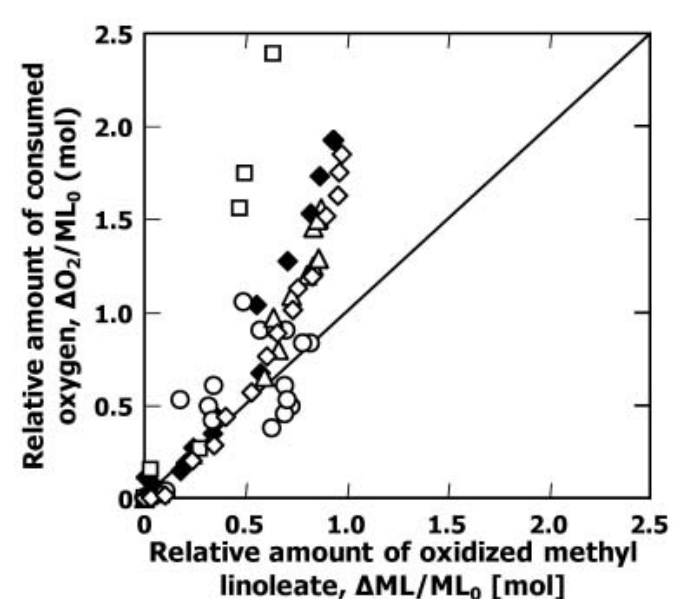

Fig. 9 Relationship between the Amount of Oxidized Methyl Linoleate and That of Oxygen Consumed during the Oxidation.

The amounts of oxidized methyl linoleate, $\Delta \mathrm{ML}$, and consumed oxygen, $\Delta \mathrm{O}_{2}$, were normalized by the initial amount of methyl linoleate, $\mathrm{ML}_{0}$. Symbols, $\bigcirc, \triangle, \square$ and $\diamond$, represent the microcapsules having oil-droplet sizes of $0.02,0.60,1.0$ and 1.0 $\mu \mathrm{m}$ in the emulsions before mixing with maltodextrin, having a weight ratio of methyl linoleate to maltodextrin of $0.2,0.35,0.2$ and 0.5 , and stored at $60,50,60$ and $50^{\circ} \mathrm{C}$, respectively. Symbol $\downarrow$ represents the oxidation of methyl linoleate in a bulk system at $40^{\circ} \mathrm{C}^{17}$.

\section{References}

1. Chakodabylu, R.; Ranajit, P.; Jagannath, G. Effect of unsaturated oils on the biosynthesis of cholesterol, and on biliary and fecal excretion of cholesterol and bile acids in rats. J. Nutr. 110, 2149-2158 (1980).

2. Harris, W.; Connor, E.; McMurry, M. The comparative reductions of the plasma lipids and lipoproteins by dietary polyunsaturated fats: Salmon oil versus vegetable oils. Metabolism 32, 179-184 (1983).

3. Matsuno, R.; Adachi, S. Lipid encapsulation technology -techniques and application to food. Trend Food Sci. Technol. 4, 256-261 (1993).

4. Ré, M. I. Microencapsulation by spray-drying. Drying Technol. 16, 1195-1236 (1998).

5. Imagi, J.; Muraya, K.; Yamashita, D.; Adachi, S.; Matsuno, R. Retarded oxidation of liquid lipids entrapped in matrixes of saccharides or proteins. Biosci. Biotechnol. Biochem. 56, 1236-1240 (1992).

6. Minemoto, Y.; Adachi, S.; Matsuno, R. Comparison of oxidation of methyl linoleate encapsulated with gum arabic by hot-air-drying and freeze-drying. J. Agric. Food Chem. 45, 4530-4534 (1997).

7. Minemoto, Y.; Hakamata, K.; Adachi, S.; Matsuno, R. Oxidation of linoleic acid encapsulated with gum arabic or maltodextrin by spray-drying. J. Microencapsulation 19, 181-189 (2002).

8. Ishido, E.; Hakamada, K.; Minemoto, Y.; Adachi, S.; Matsuno, R. Oxidation process of linoleic acid encapsulated with a polysaccharide by spray-drying. Food Sci. Technol. Res. 8, 85-88 (2002).

9. Laurent, L.; Francois, M.; Claude, G. Effect of droplet size on lipid oxidation rates of oil-in-water emulsions stabilized by protein. J. Am. Oil Chem. Soc. 79, 425430 (2002).

10. Hannnah T, O.; Casimir C, A. Effect of emulsifier type, droplet size, and oil concentration on lipid oxidation in structured lipid-based oil-in-water emulsions. Food Chem. 84, 451-456 (2004).

11. Stauffer, D. Introduction to percolation theory, Chapter 2, Taylor \& Francis, London (1985).

12. Minemoto, Y.; Adachi, S.; Matsuno, R. Autoxidation of linoleic acid encapsulated with polysaccharides of differing weight ratio. Biosci. Biotechnol. Biochem. 63, 866-869 (1999).

13. Adachi, S.; Matsuno, R. Effect of eluent composition on the distribution coefficient of saccharides onto a cation-exchange resin in sodium-ion form. Biosci. Biotechnol. Biochem. 61, 1296-1301 (1997).

14. Soottitantawat, A.; Takayama, K.; Okamura K.; Muranaka, D.; Yoshii, H.; Furuta, T.; Ohkawara, M.; Linko, P. Microencapsulation of 1-menthol by spray drying and its release characteristics. Innov. Food Sci. Emerg. Technol. 6, 163-170 (2005).

15. Soottitantawat, A.; Bigeard, F.; Yoshii, H.; Furuta, T.; Ohkawara, M.; Linko, P. Influence of emulsion and powder size on the stability of encapsulated Dlimonene by spray drying. Innov. Food Sci. Emerg. Technol. 6, 107-114 (2005).

16. Adachi, S.; Ishiguro, T.; Matsuno, R. Autoxidation kinetics for fatty acids and their esters. J. Am. Oil Chem. Soc. 72, 547-551 (1995).

17. Imai, H.; Shima, M.; Adachi, S. Oxidation of methyl linoleate in $\mathrm{O} / \mathrm{W}$ micro- and nanoemulsion systems. submitted to J. Am. Oil Chem. Soc. 